\title{
A SINGULAR STOCHASTIC INTEGRAL EQUATION
}

\author{
DAVID NUALART AND MARTA SANZ
}

\begin{abstract}
This note is devoted to the discussion of the stochastic differential equation $X d X+Y d Y=0, X$ and $Y$ being continuous local martingales. A method to construct solutions of this equation is given.
\end{abstract}

Let $(\Omega, \mathcal{F}, P)$ be a complete probability space and $\left\{\mathcal{F}_{t}, t \geq 0\right\}$ a filtration on it satisfying the usual properties. That means, $\digamma_{t}$ is right continuous, and $\digamma_{0}$ contains the null sets of $₹$.

Let $X=\left\{X_{t}, t \geq 0\right\}$ be a continuous local martingale with respect to the filtration $\left\{\xi_{t}, t \geq 0\right\}$. The continuous local martingale $M_{t}=\int_{0}^{t} X_{s} d X_{s}$ has an associated increasing process given by $\langle M\rangle_{t}=\int_{0}^{t} X_{s}^{2} d\langle X\rangle_{s}$. Denoting by $\langle M\rangle_{\text {and }}$ $\langle X\rangle$ the measures on $\mathbf{R}$ induced by the sample paths $\langle M\rangle_{t}$ and $\langle X\rangle_{t}$, respectively, we obviously have $\langle M\rangle \ll\langle X\rangle$, and $d\langle M\rangle / d\langle X\rangle=X^{2}$. Reciprocally, $\langle X\rangle \ll\langle M\rangle$, and $d\langle X\rangle / d\langle M\rangle=1 / X^{2}$. In fact, it is known that $\langle X\rangle$ does not charge the set $\{X=0\}$. have

By Itô's formula $X_{t}^{2}-X_{0}^{2}=2 M_{t}+\langle X\rangle_{t}$. So, applying the preceding result we

$$
X_{t}^{2}-X_{0}^{2}=2 M_{t}+\int_{0}^{t} \frac{1}{X_{s}^{2}} d\langle M\rangle_{s} .
$$

Therefore, a continuous local martingale $Y$ is a solution of the stochastic differential equation

$$
X d X+Y d Y=0
$$

if and only if

$$
Y_{t}^{2}-Y_{0}^{2}=-2 M_{t}+\int_{0}^{t} \frac{1}{Y_{s}^{2}} d\langle M\rangle_{s} .
$$

Equation (1) arises in a natural way in the theory of two-parameter martingales with path independent variation adapted to the $\sigma$-fields generated by two independent couples of two-dimensional brownian motions (see [4]).

First we prove a lemma that will be used to construct $Y_{t}^{2}$.

LEMMA 1. Let $b(t)$ be a continuous real function defined on $\mathbf{R}_{+}$such that $b(0) \geq$ 0 and $\mu$ a continuous measure on $\mathbf{R}_{+}$. Assume that $b(t)$ takes constant values on every interval $[a, c]$ such that $\mu([a, c])=0$. Then the integral equation

$$
r(t)=b(t)+\int_{0}^{t} \frac{1}{r(s)} d \mu_{s}
$$

has a unique, nonnegative, continuous solution.

Received by the editors July 16, 1981 and, in revised form, October 15, 1981.

1980 Mathematics Subject Classification. Primary 60H20; Secondary 60H05.

Key words and phrases. Stochastic integral, stochastic differential equations, (local) martingale. 
ProOF. The case $d \mu_{s}=d s$ was proved by McKean in [3]. In the general case define $G(s)=\inf \{t: F(t)>s\}, F$ being the distribution function of $\mu$. $G$ is right continuous and $F \circ G=$ Id.

$b \circ G$ is continuous. Indeed, fix a point $s>0$, and suppose that $G(s)=c$, $G\left(s^{-}\right)=a$. Then $F(t)=s$ for all $t$ in $[a, c]$ and, by hypothesis, $b$ is constant on $[a, c]$, which proves the continuity of $b \circ G$ on $s$.

Using McKean's result, we know that equation

$$
r^{\prime}(t)=b(G(t))+\int_{0}^{t} \frac{1}{r^{\prime}(s)} d s
$$

has a unique, nonnegative, continuous solution.

Then, if we define $r=r^{\prime} \circ F, r$ is a solution of (3). In fact,

$$
r^{\prime}(F(t))=b(G(F(t)))+\int_{0}^{F(t)} \frac{1}{r^{\prime}(s)} d s,
$$

but

$$
\int_{0}^{F(t)} \frac{1}{r^{\prime}(s)} d s=\int_{0}^{F(t)} \frac{1}{r^{\prime}(F(G(s)))} d s=\int_{0}^{t} \frac{1}{r^{\prime}(F(s))} d \mu_{s},
$$

and $b \circ G \circ F=b$ as easily follows from the assumption made on $b$.

Now we can state the main result.

THEOREM 1. Let $X$ be a continuous local martingale. Assume that there exists a sequence $\phi_{n}$ of independent random variables, with $\phi_{n} \in\{-1,1\}$ and $E\left(\phi_{n}\right)=$ 0 , such that they are independent of $X$ and $\mathcal{F}_{0}$-measurable. Then there exists $a$ continuous local martingale $Y$ such that $\int_{0}^{t}\left(X_{s} d X_{s}+Y_{s} d Y_{s}\right)=0$ for all $t \geq 0$.

ProOF. First we choose an integrable random variable $Y_{0}$ which will be the value of $Y$ at the origin.

Let us consider the equation

$$
r(t)=Y_{0}^{2}-2 M(t)+\int_{0}^{t} \frac{1}{r(s)} d\langle M\rangle_{s}
$$

where $M_{t}=\int_{0}^{t} X_{s} d X_{s}$.

It is well known (see [2]) that there exists a null set $N \subset \Omega$ such that for all $\omega \notin N,\langle M\rangle([a, c])=0$ implies that $M$ takes a constant value on the interval $[a, c]$.

So, for $\omega \notin N$ fixed, we can apply Lemma 1 to $b(t)=Y_{0}^{2}-2 M(t)$ and $\mu=\langle M\rangle$, and state the existence of a unique, nonnegative, continuous solution of (4).

Now $r(t)$ is a local submartingale because it is the sum of a local martingale and the increasing process $\int_{0}^{t}(1 / r(s)) d\langle M\rangle_{s}$. We want to show that $\sqrt{r(t)}$ also is a local submartingale. To do this, apply Itô's formula to $f(r(t))=\sqrt{r(t)+\lambda}$, where $\lambda$ is any real positive number,

$$
\begin{aligned}
\sqrt{r(t)+\lambda}= & \sqrt{r(0)+\lambda}-\int_{0}^{t}(r(s)+\lambda)^{-1 / 2} d M(s) \\
& +\int_{0}^{t} \frac{1}{2}(r(s)+\lambda)^{-1 / 2}\left(\frac{1}{r(s)}-\frac{1}{r(s)+\lambda}\right) d\langle M\rangle_{s} .
\end{aligned}
$$

Let $\left\{T_{n}, n \in N\right\}$ be an increasing sequence of stopping times such that $T_{n} \uparrow \infty$ and $M^{T_{n}}$ is a martingale bounded by $n$. Set $R_{n}=\inf \{t, r(t) \geq n\}$ and $S_{n}=T_{n} \wedge$ 
$R_{n}$. Then $\sqrt{r\left(t \wedge S_{n}\right)+\lambda}$ is a positive submartingale, and letting $\lambda$ tend to zero we obtain, by monotone convergence, the submartingale property of $\sqrt{r\left(t \wedge S_{n}\right)}$. Therefore $\sqrt{r(t)}$ is a local submartingale because $S_{n} \uparrow \infty$.

The increasing process given by the Doob decomposition of $\sqrt{r(t)}$ is $A_{t}=$ $\sqrt{r(t)}-\sqrt{r(0)}+\int_{0}^{t}(r(s))^{-1 / 2} d M(s)$. In fact, for any $\lambda>0, \sqrt{r\left(t \wedge S_{n}\right)+\lambda}-$ $\sqrt{r(0)+\lambda}+\int_{0}^{t \wedge S_{n}}(r(s)+\lambda)^{-1 / 2} d M(s)$ is increasing.

We next show that $\int_{0}^{t} 1_{\{\sqrt{r(s)}>0\}} d A_{s}=0$. Indeed, we have

$$
\begin{aligned}
r(t)= & \left(A_{t}+\sqrt{r(0)}\right)^{2}-2\left(A_{t}+\sqrt{r(0)}\right) \int_{0}^{t}(r(s))^{-1 / 2} d M(s) \\
& +\left(\int_{0}^{t}(r(s))^{-1 / 2} d M(s)\right)^{2}
\end{aligned}
$$

Computing the bounded variation part of each term we obtain

$$
\begin{aligned}
r(0)+\int_{0}^{t} \frac{1}{r(s)} d\langle M\rangle_{s}= & \left(A_{t}+\sqrt{r(0)}\right)^{2}-2 \int_{0}^{t}\left(\int_{0}^{s}(r(u))^{-1 / 2} d M(u)\right) d A_{s} \\
& +\int_{0}^{t} \frac{1}{r(s)} d\langle M\rangle_{s} .
\end{aligned}
$$

Therefore,

$$
0=\left(A_{t}+\sqrt{r(0)}\right)^{2}-r(0)-2 \int_{0}^{t}\left(\int_{0}^{s}(r(u))^{-1 / 2} d M(u)\right) d A_{s}=2 \int_{0}^{t} \sqrt{r(s)} d A_{s},
$$

which implies the assertion.

Henceforth, for any $n \geq 1,\left\{\rho_{n}(t)=\sqrt{r\left(S_{n}+t\right) \wedge S_{n+1}}, \mathcal{F}_{S_{n}+t}, t \geq 0\right\}$ is a submartingale which satisfies $\int_{0}^{t} 1_{\left\{\rho_{n}(s)>0\right\}} d A_{s}^{n}=0$, where

$$
A_{t}^{n}=A\left(\left(S_{n}+t\right) \wedge S_{n+1}\right)
$$

is the increasing process associated to $\rho_{n}(t)$. Using Barlow's procedure (see [1]) it is possible to find a martingale $M_{n}(t)$ such that $\left|M_{n}(t)\right|=\rho_{n}(t)$ and $M_{n}(0)=$ $M_{n-1}\left(S_{n}\right)$ (we take $S_{0}=0$ and $M_{0}=Y_{0}$ ). Then $Y=\sum_{n} M_{n} 1_{\llbracket T_{n}, T_{n+1} \rrbracket}$ is a nonnegative local submartingale whose absolute value is $\sqrt{r(t)}$. Note that according to Barlow's method, the sign of $Y$ is defined in terms of the sequence $\phi_{n}$. So $Y_{t}^{2}=$ $r(t)$ satisfies equation (2) and this finishes the proof of the theorem.

REMARKS. 1. Instead of assuming that the $\sigma$-field $\digamma_{0}$ is rich enough to contain the sequence $\phi_{n}$, we can adjoin a new probability space and show the existence of the local martingale $Y$ in the product space.

2. There is no uniqueness of the solution because $-Y$ also is a solution. Moreover the random variable $Y_{0}$ is arbitrary.

3. Let $T=\inf \left\{t, Y_{t}=0\right\}$. If the initial value $Y_{0}$ is given, there is uniqueness of the solution in $[0, T]$. Furthermore, to construct the solution in this interval we do not need the sequence $\phi_{n}$.

4. Suppose that $Y_{0}=X_{0}$. In this case, the processes $X^{2}$ and $Y^{2}$ may have the same law. A sufficient condition for it to hold is the equality between the law of $\left\{\left(M_{t},\langle M\rangle_{t}\right), t \geq 0\right\}$ and that of $\left\{\left(-M_{t},\langle M\rangle_{t}\right), t \geq 0\right\}$. For example, this condition holds if $M_{t}$ is a Wiener process. 
5. As a consequence of Theorem 1 we obtain the existence of nonnull twodimensonal martingales with increasing norm.

\section{REFERENCES}

1. M. T. Barlow and M. Yor, Sur la construction d'une martingale continue d valeur absolue donnée, Séminaire de Probabilités XIV (62-75), Lecture Notes in Math., vol. 784, Springer-Verlag, Berlin and New York, 1980.

2. R. Getoor and M. Sharpe, Conformal martingales, Invent. Math. 16 (1972), 271-308.

3. H. P. McKean, The Bessel motion and a singular integral equation, Mem. Coll. Sci. Univ. Kyoto Ser. A 33 (1960), 317-322.

4. D. Nualart, Martingales d̀ variation indépendante du chemin, Proc. Aléatoires à Deux Indices, Proceedings, Paris, 1980, Lecture Notes in Math., vol. 863, Springer-Verlag, Berlin and New York, 1981.

Departament d'Estadística, Facultat de Matemàtiques, Universitat de BARCELONA, BARCELONA, SPAIN

Departament d'Estadística, Facultat de Ciències, Universitat Autónoma DE BARCElona, Bellaterra (BARCELona), SPAin 